\title{
Development of a Web Database System for a Comprehensive Hypertension Management Program in the Primary Health Care Setting of Urban Areas of Peru
}

\author{
Hye Yeon Jo ${ }^{1,2}$, Jorge A. Estrada Vidal ${ }^{3}$, Oscar O. Giraldo Castillo ${ }^{3}$, Luis A. Mayta Mamani ${ }^{3}$, \\ Eun Woo Nam ${ }^{1,2}$ \\ ${ }^{1}$ Department of Health Administration, College of Health Science, Yonsei University, Wonju, Korea \\ ${ }^{2}$ Yonsei Global Health Center, Wonju, Korea \\ ${ }^{3}$ DGNEST, Lima, Peru
}

Objectives: The objective of this case report is to introduce the development process, structure, characteristics, and effectiveness of the web database (DB) system developed for the hypertension management program in the primary health care setting in the low-income urban areas of Peru. Methods: A Korean research team cooperated with a Peruvian IT startup company to develop a web DB system to improve the data management of the hypertension management program. The entire web application infrastructure was hosted using the Amazon Web Service. Two different web platforms were established for the user groups of health workers and researchers (program managers). Results: A total of 2,827 program participants were registered in the web DB system until December 2017. Health professionals can input the participant data while providing consultation to the program participants, and the data is accumulated in the web DB in real time. Input errors or data loss is prevented by setting restrictions in the data entry system. During the 4-year project period, 7,696 hours of working time and USD 39,536.48 for data management were saved as the result of web DB system utilization. Conclusions: The developed web DB system contributed to improve the health condition of the health program participants by providing necessary information to the health professionals at the right time. This case report could be a reference for other researcher to develop web databases for their own context, especially in developing countries.

Keywords: Health Information System, Electronic Health Records, Primary Health Care, Developing Countries, Peru

Submitted: June 24, 2018

Revised: 1st, August 3, 2018; 2nd, January 15, 2019

Accepted: January 25, 2019

\section{Corresponding Author}

Eun Woo Nam

Department of Health Administration, College of Health Science, Yonsei University, 1 Yeonsedae-gil, Wonju 26493, Korea. Tel: +82-33-7602573, E-mail: ewnam@yonsei.ac.kr (https://orcid.org/0000-0001-6584-0658)

This is an Open Access article distributed under the terms of the Creative Commons Attribution Non-Commercial License (http://creativecommons.org/licenses/by-nc/4.0/) which permits unrestricted non-commercial use, distribution, and reproduction in any medium, provided the original work is properly cited. 


\section{Introduction}

The health information system provides the foundation of decision making in public health by providing appropriate evidence-based information [1]. Electronic Health Records (EHRs) can contribute to the better management of health information and improve the quality of health services [2-4]. Although middle- and low-income countries are facing a great need for EHRs utilization [5,6], not enough related practices have been implemented, including countries in Latin America [7,8].

With the goal of promoting the health of community populations in low-income urban areas in Peru, the "Comprehensive Hypertension Management Program" was conducted from May 2015 to December 2017, as a health official development assistance (ODA) project.

The data management of this ODA project was handled through a paper-based system for the first year of implementation, and we faced difficulties with this method. Health personnel needed to work overtime for data management, and time delays occurred in database construction. Some data were missing, and human errors lowered the quality of the dataset.

We decided to develop a web database (DB) system to solve the problems mentioned above. The objective of this case report is to introduce the development process, structure, characteristics, and effectiveness of the web DB system developed for the hypertension management program in the primary health care setting of the low-income urban areas of Peru.

\section{Case Description}

\section{The Process of System Development}

We utilized the Scrum methodology for the web DB system development process. Scrum is an iterative and incremental agile software development framework. The development period was a total of 4 months from July to October 2016.

All Scrum workflow is based on sprints; each sprint starts with a sprint planning event that aims to define a sprint backlog, identify the sprint work and establish an estimated commitment to the sprint goal. Each sprint ends with a sprint review and retrospective to show to stakeholders who review the progress and identify lessons and improvements for the next sprint. In this project, we used four sprints of 2 to 3 weeks each.

Aiming to optimize the web DB system to satisfy the needs of the project field, we provided presentations of system utilization to the target users, including local health professionals and project managers, during the system development period, and received their feedback to supplement the system.

\section{System Infrastructure and Characteristics}

The entire web application infrastructure is hosted using the Amazon Web Server on Linux. The NGINX web server uses uWSGI and the DJANGO framework with Python for the server-side to interpret the programming language. We automated the infrastructure using Ansible to deploy as many web application servers as needed. In addition, we exposed a RESTful API which can be applied for both web and mobile applications.

All data are stored in the POSTGRES relational database. In addition, all asynchronous tasks are conducted by the brokers Celery and RabbitMQ (Figure 1).

For the front-end side, a web application was developed as a 'single-page application' because the page is retrieved with a single-page load for the browser using this type of web application. Then everything was retrieved dynamically by asynchronous requests to the server, increasing the load time speed of all views, thus achieving a good user experience.

Therefore, the web application is responsive, adapting to the screen size of any device, such as a desktop, laptop, smartphone, tablet, for the contents to be properly visualized.

\section{Platform for System Users}

System users can be classified into two groups: health workers and researchers. Two different web platforms were established for the user groups.

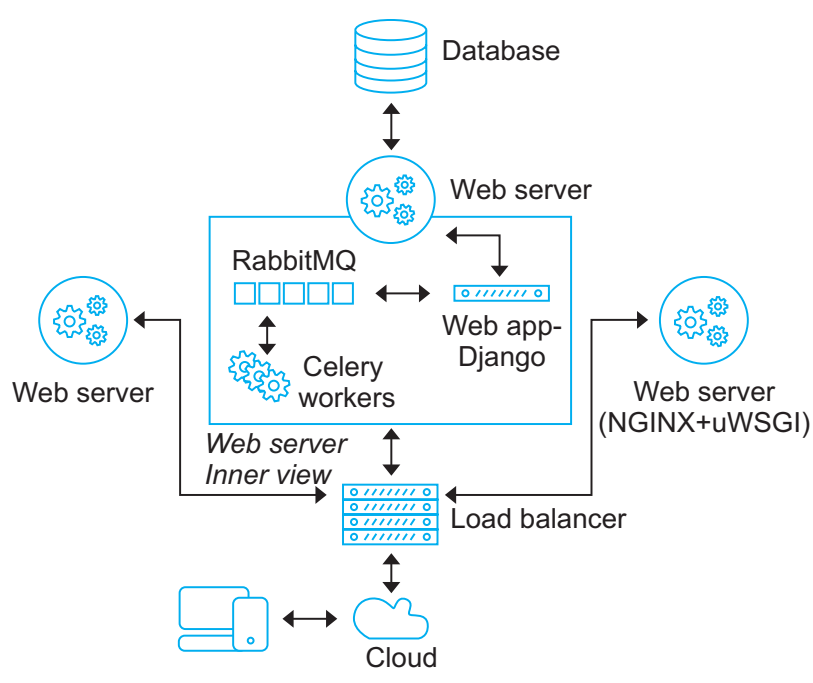

Figure 1. System diagram. 
Through the platform for health workers, health professionals can input and save the data of program participants while providing consultation to them (Figures 2-4). The participant data is recorded and accumulated in the web DB in real time.

Before the web DB system was developed, health workers entered the same data two times, once using a paper chart and a second time using an electronic document. Since the system was developed, data entry is performed only one time through the webpage.

In the platform for researchers, the researchers can access the database and download the dataset in an electronic document format in the 'exportable' section page (Figure 5).

With the former paper-based system, a data manager checked the entry errors or missing data in the dataset. When any problematic record was observed, a correction was requested from health workers. In contrast, after the web DB system was developed, input errors or data loss could be prevented by setting restrictions in the data entry system. For example, we set a range of possible numbers to enter into the section of 'height', from 130 to 200. Numerical values that are not included in the designated range are not allowed to be input on the entry page.

\section{Effectiveness of Using the Web DB System}

During the 4 years of the entire project period, the time for data management was reduced through use of the web DB system by 7,696 hours.

To calculate the labor cost of data management, we referred to the wages and working hours of the health personnel in the project area of Peru in 2016, when the number of newly registered program participants was an average of 100 per
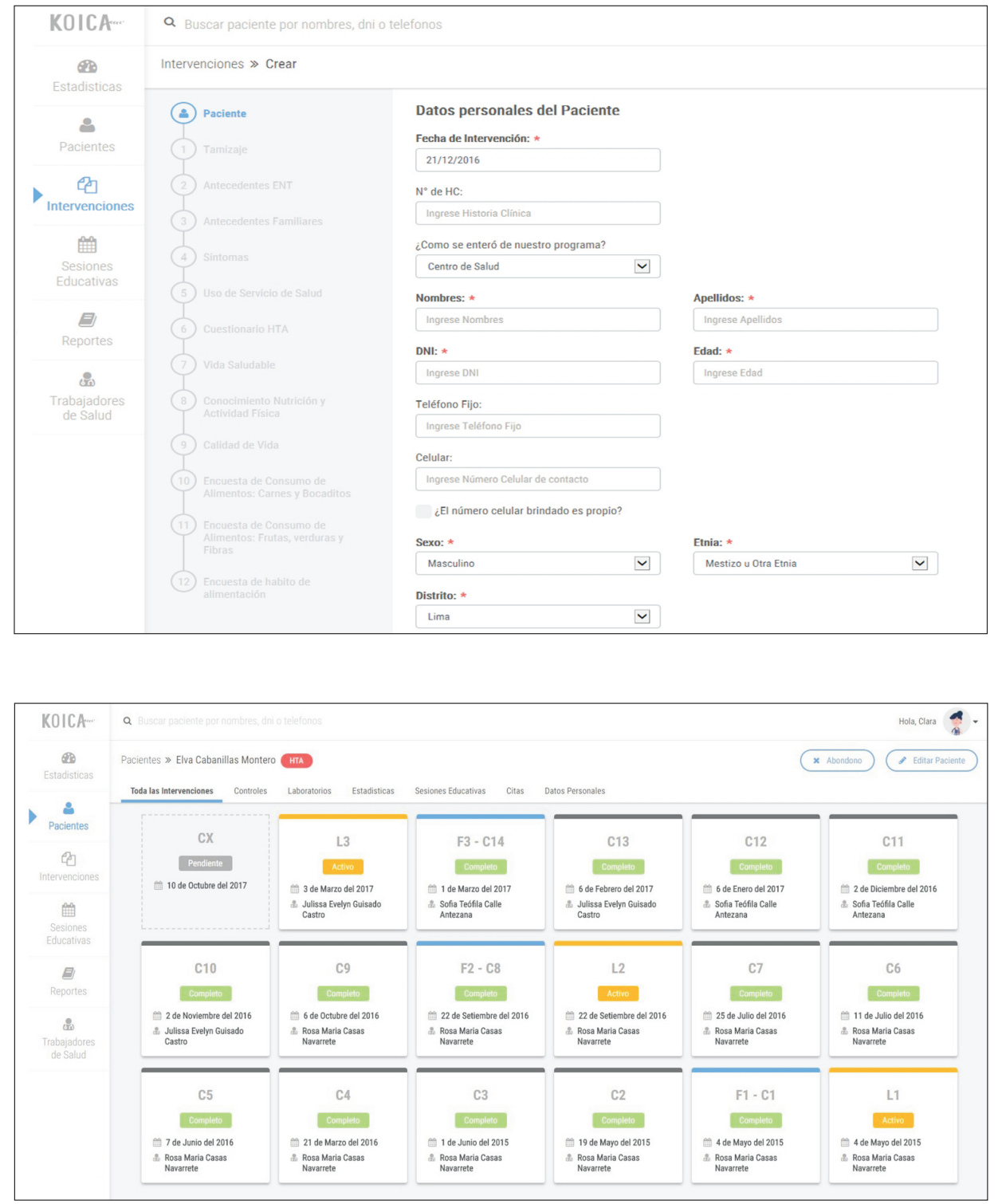

Figure 2. Health worker module platform (data entry page).
Figure 3. Patient section for reviewing previous interventions and generating records of a new intervention. 

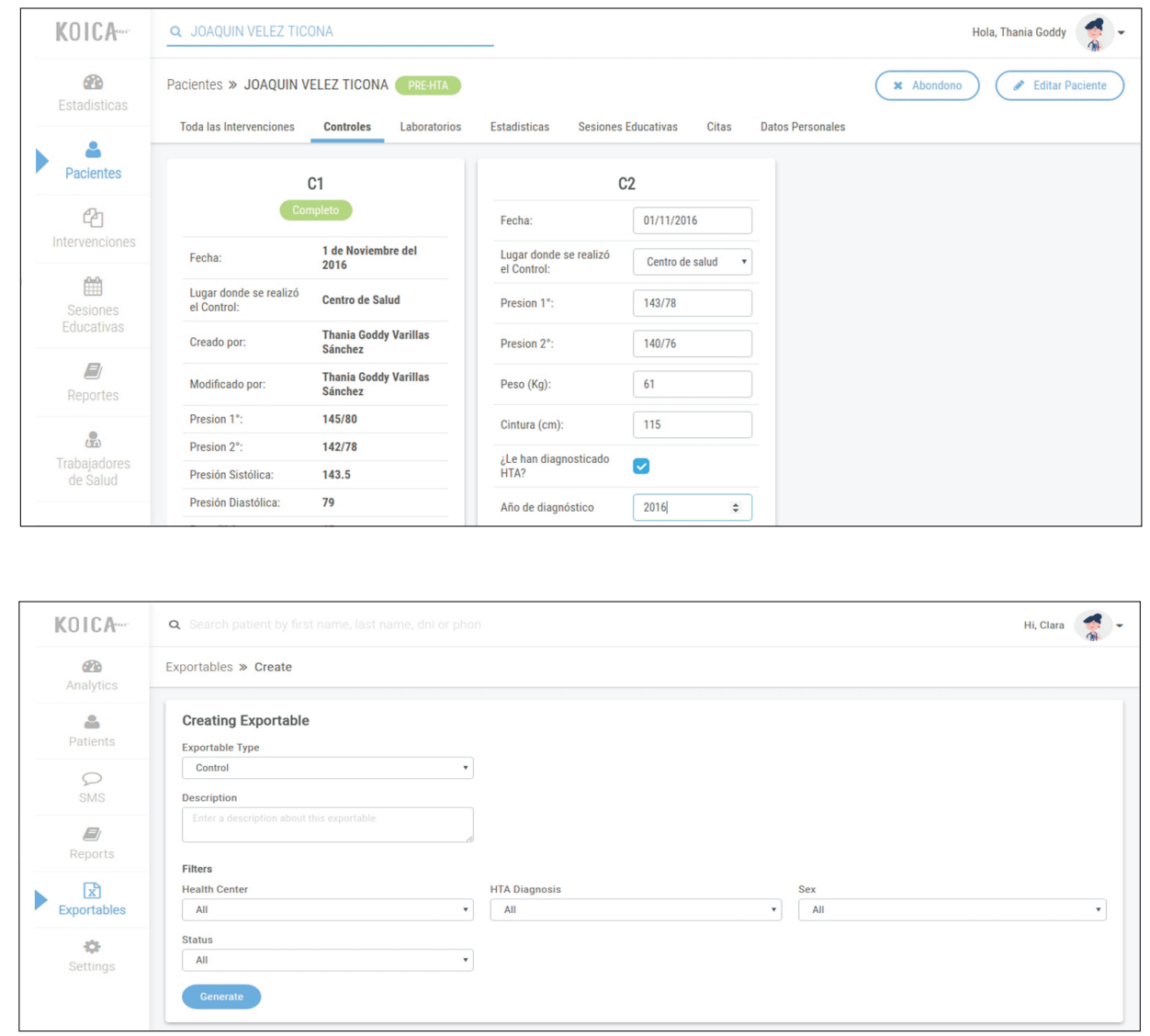

Figure 4. Patient section for creating a new individual consultation record.

Figure 5. Exportable section for generating a new exportable dataset file.

Table 1. Reductions of working time and labor cost by using the web database system

\begin{tabular}{|c|c|c|c|c|c|}
\hline \multirow{2}{*}{ System } & \multirow{2}{*}{ Personnel } & \multicolumn{2}{|c|}{ Labor cost (USD/person) } & \multirow{2}{*}{ Total time $^{\mathrm{a}}(\mathrm{hr})$} & \multirow{2}{*}{ Total labor cost (USD) } \\
\hline & & Monthly & Hourly & & \\
\hline \multirow[t]{3}{*}{ Paper based } & Health worker & 690 & 4.11 & 936 & $3,846.96$ \\
\hline & Data manager & 850 & 6.07 & 1,092 & $6,628.44$ \\
\hline & Total & & & 2,028 & $10,475.40$ \\
\hline Web database & Data manager & 850 & 6.07 & 104 & 631.28 \\
\hline \multicolumn{2}{|c|}{ Difference (reduced amount) } & & & 1,924 & $9,844.12$ \\
\hline
\end{tabular}

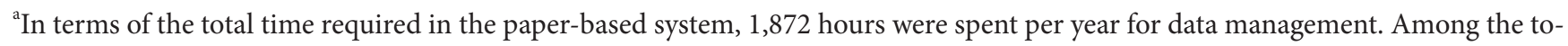
tal hours, 864 hours ( 6 hours $\times 52$ weeks $\times 3$ health centers) were spent for data input, 546 hours $(10.5$ hours $\times 52$ weeks) for tracking and correcting errors, 364 hours ( 7 hours $\times 52$ weeks $\times 12$ months) for data cleaning, and 182 hours ( 3.5 hours $\times 52$ weeks) for merging datasets and constructing the database. In comparison, only time for data cleaning is needed with the web database system because data are automatically entered by the system. For data cleaning, a total of 104 hours ( 2 hours $\times 52$ weeks) were required per year.

month, or 1,200 per year.

A total of USD 10,475.40 was needed for the paper-based system for 1 year, while USD 631.28 was spent with the web DB system, showing that the web DB system reduces the labor cost by USD 9,844.12 per year (Table 1).

In the paper-based system, the labor cost of additional working hours was needed, while the cost of system devel- opment and maintenance was necessary for using the web DB system. We compared the costs of the two different data management methods.

A total of USD 9,000 was spent for the development of the web DB system, and USD 840 per year was spent as the system maintenance cost. Specific details of expenses are presented in Table 2. Considering the development and 
Table 2. Cost of web database system maintenance

\begin{tabular}{lcc}
\hline \multirow{2}{*}{ System maintenance } & \multicolumn{2}{c}{ Cost (USD) } \\
\cline { 2 - 3 } & Yearly & Monthly \\
\hline Web server & 480 & 40 \\
Database server & 120 & 10 \\
Load balancer server & 120 & 10 \\
Scheduler server & 120 & 10 \\
Total & 840 & 70 \\
\hline
\end{tabular}

maintenance cost of the web DB system and the labor cost reduction achieved through introduction of the system, the economic benefit generated after 13 months of utilization of the web DB system exceeded the amount of investment.

During the 4-year project period, the cost reduction for data management by using the web DB system was a total of USD 39,536.48.

\section{Discussion}

This study aims to describe the structure and characteristics of the web DB system developed for constructing a participants' database of the hypertension program in the primary healthcare setting of the urban areas of Peru. All program participants were registered in the web DB system (a total of 2,827 participants in December 2017).

According to a professional physician who worked as the field manager of the hypertension management program for 2 years (from 2015 to 2016), the web-based system enabled various health professionals, including doctors, nurses, and nutritionists, to easily monitor the participants' information and to provide proper interventions to address the condition of each participant.

The physician also mentioned that the assessment of the appropriate drug treatment and medication adherence was facilitated by monitoring anthropometric indicators, including blood pressure (BP), body mass index (BMI), waist circumference, glucose, and creatinine, though the web DB system, and this allows an actual decrease in BP, improvement of the obesity index, and the treatment of comorbid diseases, such as hyperlipidemia.

At the beginning of this project, challenges existed regarding EHRs in Peru. There had been almost no EHR system for patient registration and management in the primary healthcare setting of Peru $[9,10]$. When we interviewed public officers in the health information sector of Peruvian regional government, they mentioned the struggles involved in establishing national health statistics due to paper-based health records in Peru. The officers also recognized the necessity of a nationwide introduction of information technology for efficient data management.

Furthermore, we can also refer the cases of the web DB applications being utilized in other countries, such as the web DB for World Health Organization healthy city Wonju in the Republic of Korea. This is a web DB application established for the purpose of systematically computing the health indicators relevant to the healthy city project $[11,12]$.

We expect that the introduction of health information technology will improve the quality of health services for communities in developing countries, and we hope that this report will be helpful for that improvement.

\section{Conflict of Interest}

No potential conflict of interest relevant to this article was reported.

\section{Acknowledgments}

This work was supported by the Korea International Cooperation Agency (KOICA) under the title of "Health Promotion Program in Northern Lima and Callao, Peru” in 2013 (No. P2013-00151-1).

\section{ORCID}

Hye Yeon Jo (http://orcid.org/0000-0002-0697-5576)

Jorge A. Estrada Vidal (http://orcid.org/0000-0002-0081-1829)

Oscar O. Giraldo Castillo (http://orcid.org/0000-0002-6589-4645)

Luis A. Mayta Mamani (http://orcid.org/0000-0003-0759-5687)

Eun Woo Nam (http://orcid.org/0000-0001-6584-0658)

\section{References}

1. World Health Organization. Framework and Standards for country health information systems. 2nd ed. Geneva, Switzerland: World Health Organization; 2008.

2. Varshney U. Pervasive healthcare computing: EMR/ EHR, wireless and health monitoring. New York (NY): Springer; 2009.

3. Jones SS, Rudin RS, Perry T, Shekelle PG. Health information technology: an updated systematic review with a focus on meaningful use. Ann Intern Med 2014;160(1):48-54.

4. Chaudhry B, Wang J, Wu S, Maglione M, Mojica W, Roth E, et al. Systematic review: impact of health infor- 
mation technology on quality, efficiency, and costs of medical care. Ann Intern Med 2006;144(10):742-52.

5. Fritz F, Tilahun B, Dugas M. Success criteria for electronic medical record implementations in low-resource settings: a systematic review. J Am Med Inform Assoc 2015;22(2):479-88.

6. Hossain N, Yokota F, Sultana N, Ahmed A. Factors Influencing Rural End-Users' Acceptance of e-Health in Developing Countries: A study on Portable Health Clinic in Bangladesh. Telemed J E Health 2018 Apr 17 [Epub]. http://doi.org/10.1089/tmj.2018.0039.

7. Program for Appropriate Technology in Health (PATH). An interim review of the Health Information Systems Programme (University of Oslo) with recommendations for future action. Seattle (WA): PATH; 2016.

8. Giovanella L, Feo O, Faria M, Tobar S. Health systems in South America: challenges to the universality, integrality and equity. Rio de Janeiro, Brazil: South American
Institute of Governance in Health (ISAGS); 2013.

9. Perez-Lu JE, Iguiniz Romero R, Bayer AM, Garcia PJ. Wawared Peru: reducing health inequities and improving maternal health by improving information systems in health. Rev Peru Med Exp Salud Publica 2015;32(2):373-7.

10. Blaya JA, Cohen T, Rodriguez P, Kim J, Fraser HS. Personal digital assistants to collect tuberculosis bacteriology data in Peru reduce delays, errors, and workload, and are acceptable to users: cluster randomized controlled trial. Int J Infect Dis 2009;13(3):410-8.

11. Nam EW, Shin TS, Song YL, Park KS, Song TM, Kim $\mathrm{MK}$, et al. Building web database for WHO healthy city Wonju. Korean J Health Educ Promot 2007;24(3):11928.

12. Nam EW, Park JS, Choe EH, Kim GN. A study on the application of web database for healthy city Wonju. Korean J Health Serv Manag 2012;6(1):219-29. 